\title{
Effect of transgenic GDNF expression on gentamicin- induced cochlear and vestibular toxicity
}

\author{
M Suzuki ${ }^{1,2}$, M Yagi ${ }^{1,3}$, JN Brown ${ }^{1}$, AL Miller $^{1}$, JM Miller $^{1}$ and Y Raphael ${ }^{1}$ \\ ${ }^{1}$ Kresge Hearing Research Institute, The University of Michigan, Ann Arbor, MI, USA; ${ }^{2}$ Department of Otolaryngology, University \\ of Tokyo, Tokyo; and ${ }^{3}$ Department of Otolaryngology, Kansai Medical University, Osaka, Japan
}

\begin{abstract}
Gentamicin administration often results in cochlear and/or vestibular hair cell loss and hearing and balance impairment. It has been demonstrated that adenovirus-mediated overexpression of glial cell line-derived neurotrophic factor (GDNF) can protect cochlear hair cells against ototoxic injury. In this study, we evaluated the protective effects of adenovirus-mediated overexpression of GDNF against gentamicin ototoxicity. An adenovirus vector expressing the human GDNF gene (Ad.GDNF) was administered into the scala vestibuli as a rescue agent at the same time as gentamicin, or as a protective agent, 7 days before gentamicin
\end{abstract}

administration. Animals in the Rescue group displayed hearing thresholds that were significantly better than those measured in the Gentamicin or Ad.LacZ/Gentamicin groups. In the Protection group, Ad.GDNF afforded significant preservation of utricular hair cells. The data demonstrated protection of the inner ear structure, and rescue of the inner ear structure and function against ototoxic insults. These experiments suggest that inner ear gene therapy may be developed as a clinical tool for protecting the ear against environmentally induced insults. Gene Therapy (2000) 7, 1046-1054.

Keywords: adenovirus; gene transfer; GDNF; gentamicin; cochlea; utricle

\section{Introduction}

Neurotrophins are macromolecules that affect development and survival of neurons in the central and peripheral nervous systems. ${ }^{1,2}$ In the cochlea, neurotrophic factors such as brain-derived neurotrophic factor (BDNF) and neurotrophin-3 (NT-3) have been found in hair cells during development ${ }^{3}$ and in the mature organ. ${ }^{4}$ In the vestibular neuro-epithelium, BDNF mRNA has been found in hair cells of the immature and mature inner ear. ${ }^{4}$ In NT-3 and BDNF mutant mice, vestibular type II hair cells have been found to be morphologically different from those observed in normal mice. ${ }^{1}$ Overexpression of BDNF has been shown to protect vestibular hair cells from degeneration due to ototoxic drugs. ${ }^{5}$

Glial cell line-derived neurotrophic factor (GDNF) is a distantly related member of the transforming growth factor- $\beta$ (TGF- $\beta$ ) superfamily. GDNF is a potent survival factor for midbrain dopaminergic, spinal motor, cranial sensory and sympathetic neurons. ${ }^{6-8}$ GDNF mRNA is expressed in some tissues receiving sympathetic innervation, such as kidney, stomach, lung and salivary gland. ${ }^{8}$ In the inner ear, Nosrat et al ${ }^{9}$ have reported that GDNF mRNA expression was seen in the spiral ganglion and the sensory epithelium of the developing inner ear. GDNF mRNA expression was also found in mammalian hair cells during development and in the mature organ of Corti, ${ }^{10}$ suggesting a potential role of GDNF in the

Correspondence: Y Raphael, Room 9303, MSRB III, The University of Michigan, Ann Arbor, MI 48109-0648, USA

Received 12 August 1999; accepted 5 February 2000 developing and mature cochlea. mRNAs of GDNF receptors alpha and RET, the two receptors that are indispensable for GDNF action, have been detected in the organ of Corti of mature rats. ${ }^{11}$ Infusion of GDNF into the inner ear via mini-osmotic pumps has been shown to reduce cochlear hair cell lesions caused by intensive noise in guinea pigs. ${ }^{12,13}$ The effect of GDNF overexpression on the susceptibility of vestibular hair cells to experimentally induced insults has not been determined.

Virus-mediated gene transfer is a powerful technique employed to efficiently express transgenes in cells and tissues. ${ }^{14-16}$ Overexpression of transgenes can be used for protection, rescue from trauma, or correction of genetic defects. In the inner ear, several viral vectors have been used for delivering transgenes. These vectors include herpes simplex virus, adenovirus, adeno-associated virus and vaccinia virus. ${ }^{17-20}$ It has been demonstrated that adenovirus-mediated overexpression of GDNF can protect cochlear hair cells from cochleotoxicity induced by systemic administration of kanamycin and ethacrynic acid. $^{21}$ However, the effects of virus-mediated GDNF transgene expression for prevention of aminoglycosideinduced vestibulotoxicity have not been determined.

Gentamicin, derived from the species Actinomyces micromonospora, was released for clinical use in the early 1960 s. $^{22}$ Gentamicin is generally used for the treatment of severe gram-negative infections. Its most severe sideeffects include nephrotoxicity as well as toxicity in the cochlear and vestibular neuro-epithelium. ${ }^{22,23}$ Interventions for prevention of gentamicin-induced inner ear toxicity are necessary, but clinically unavailable at this time. In addition to fighting bacterial infections, gentamicin is often used to eliminate vestibular function for 
relieving vertigo and tinnitus in patients suffering from Meniere's disease and other debilitating vestibular dysfunctions. In order to preserve the cochlear hair cells and the hearing in these patients, it is necessary to restrict the action to the vestibular organs. Such selective vestibular lesions are not currently possible.

The purpose of this study is to determine the effects of adenovirus-mediated GDNF transgene expression on cochlear and vestibular hair cells exposed to gentamicin. The data demonstrate efficient rescue of vestibular hair cells, auditory hair cells, and auditory thresholds in ears inoculated with the GDNF vector.

\section{Results}

\section{Distribution of reporter transgene}

In animals in the Ad.LacZ Control group, large numbers of blue nuclei were observed in the vestibular and auditory areas of the inner ear. In the utricle, cells with blue nuclei were located in the layer of connective tissue beneath the epithelial sheet (Figure 1). In the cochlea, most transduced cells were observed among mesothelial cells of the scala vestibuli in the basal turn of the cochlea, as previously demonstrated. ${ }^{24}$

\section{General response to surgery and hearing function}

Following recovery from general anesthesia, all animals had a head tilt to the side of the surgery (ipsilateral). The head tilt disappeared within $24 \mathrm{~h}$ in all but one animal, in which it was resolved in 2 days.

Auditory brainstem response (ABR) threshold levels before and after injection were compared for the Vehicle and Ad.LacZ Control groups, using a two-way repeated measures analysis of variance. There were no statistically significant differences in threshold levels between groups or between time-points for the three frequencies assessed (Figure 2). In addition, a two-way repeated measures analysis of variance found no significant differences between the threshold shifts (after injection minus before injection) for the two groups, at the frequencies assessed.

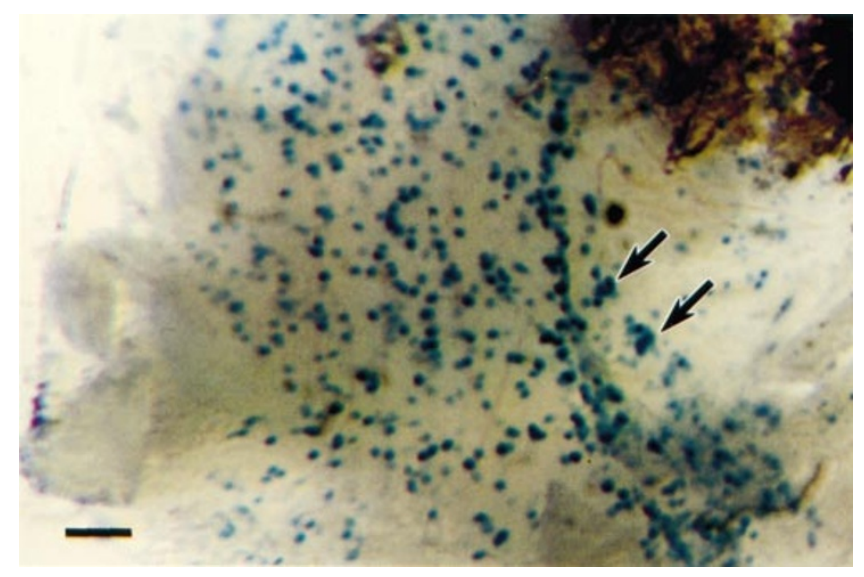

Figure 1 A stereoscopic micrograph showing the distribution of LacZpositive cells in an utricle from the Ad.LacZ Control group. Blue dots represent nuclei of cells expressing the LacZ transgene. LacZ-positive cells are found in the area immediately beneath the utricular macula and in adjacent connective tissue surrounding the edge of the utricle. The arrows point at the circular edge of the disk-shaped utricular macula. The highest density of transgene expression is in the connective tissue attached to the non-sensory portion of the utricle (to the left of the arrows). Bar, $35 \mu \mathrm{m}$.

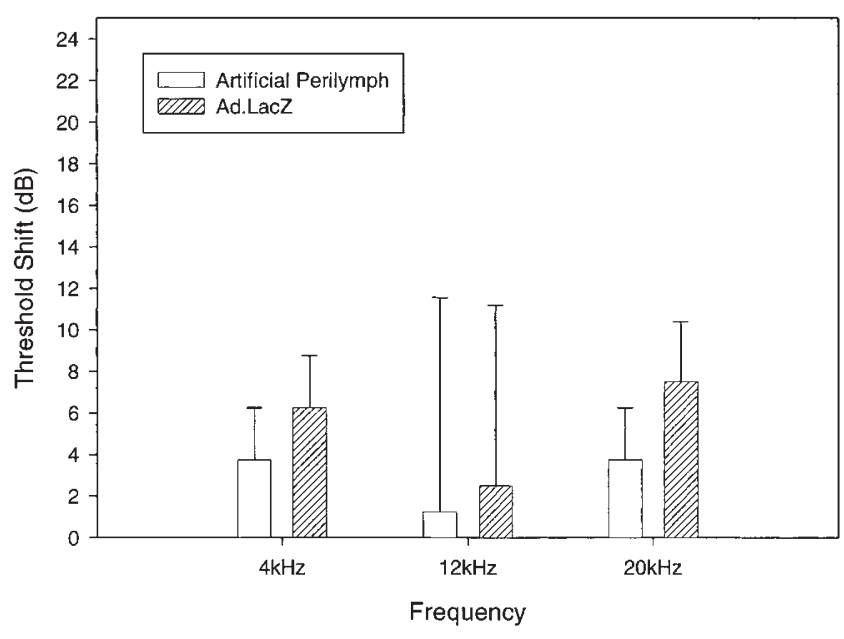

Figure $2 A B R$ threshold shifts (mean \pm s.d.) at each frequency tested for the Vehicle Control and the Ad.LacZ Control groups. No statistically significant difference of hearing thresholds is observed at any frequency between pre-and post-inoculation, nor is there a significant difference in threshold shift between groups.

All animals in the Gentamicin group showed severe threshold shifts (Figure 3). In two of these animals, no $\mathrm{ABR}$ waves could be detected at any frequency tested in response to an $85 \mathrm{~dB}$ SPL stimulus before killing the animals. Five animals in the Ad.LacZ/Gentamicin group showed various threshold shifts at all frequencies tested (Figure 3 shows mean threshold shifts of the Ad.LacZ/Gentamicin group). One of the five animals showed no response at $12 \mathrm{kHz}$. In another animal, there was no response to $12 \mathrm{kHz}$ and $20 \mathrm{kHz}$ stimuli.

Of the eight animals in the Rescue group, seven animals showed nearly normal ABR thresholds at $4 \mathrm{kHz}$ and

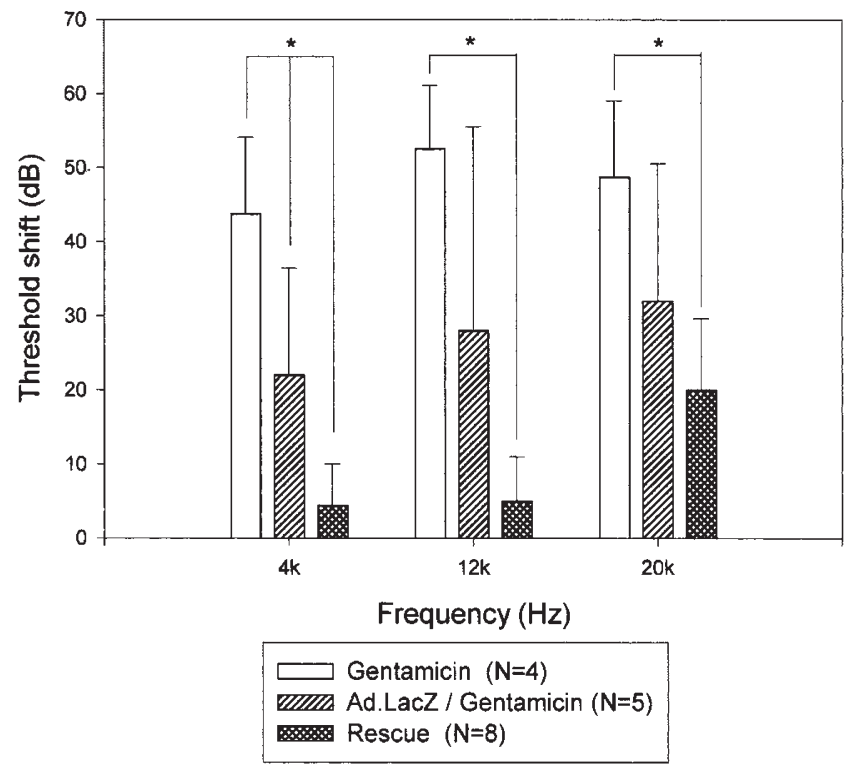

Figure 3 ABR threshold shifts (mean \pm s.d.) observed among Gentamicin, Ad.LacZ/Gentamicin and Rescue animals. A significant difference of threshold shift at $4 \mathrm{kHz}$ is found between the Gentamicin, Ad.LacZ/Gentamicin and Rescue groups. Threshold shifts at 12 and $20 \mathrm{kHz}$ in the Rescue group are significantly different from those in the Gentamicin group. *Significant difference $(P<0.05)$. 
$12 \mathrm{kHz}$. The eighth animal in this group showed $15 \mathrm{~dB}$ threshold shifts at $4 \mathrm{kHz}$ and $12 \mathrm{kHz}$. Measurements at $20 \mathrm{kHz}$ showed 15-40 dB threshold shifts in six of the eight animals. The remaining two animals maintained normal hearing. A statistically significant difference was found among the threshold shifts obtained at $4 \mathrm{kHz}$ in the Gentamicin, Ad.LacZ/Gentamicin and Rescue groups (Figure 3). Hearing threshold shifts at $12 \mathrm{kHz}$ and $20 \mathrm{kHz}$ were significantly smaller in the Rescue group as compared with those in the Gentamicin group. The differences of threshold shifts at $12 \mathrm{kHz}$ and $20 \mathrm{kHz}$ between Gentamicin and Ad.LacZ/Gentamicin groups were not significant. However, there was a trend for better thresholds in the Ad.LacZ/Gentamicin group (Figure 3).

\section{Inner and outer hair cell loss in the cochlea}

In the Vehicle Control and Lac-Z Control groups, mean inner and outer hair cell loss was about $1 \%$ or less in all animals following inoculation (Figure 4). No statistically significant difference was found in inner and outer hair cell loss in the Vehicle Control group as compared with the Lac-Z Control group. In the hook portion (the most basal portion of the cochlear duct), it was not possible to obtain complete preparations for reliable quantification. Qualitative examination of the hooks that were available revealed hair cell loss in animals in Gentamicin, Ad.LacZ/Gentamicin, Rescue and Protection groups.

Severe loss of the inner and outer hair cells was observed in all turns of the cochlea in the Gentamicin group (Figure 4). The extent of hair cell loss among the five animals in the Ad.LacZ/Gentamicin group was variable. The mean hair cell loss in the Ad.LacZ/Gentamicin group is plotted in Figure 5. Three of the animals showed severe outer hair cell loss in the basal portion of the cochlea. In two of these three animals, the lesion extended to the apical cochlea. The other two animals in the Ad.LacZ/Gentamicin group had nearly complete preservation of the inner and outer hair cells throughout the cochlear duct. In Rescue group animals, the inner and outer hair cells were well preserved in the upper basal to apical turns (Figure 5). The lower portion of the basal turn demonstrated a variable degree of hair cell loss in Rescue group animals.

Epi-fluorescence images of rhodamine phalloidinstained whole-mount preparations of the organ of Corti showed that in the Rescue group inner and outer hair cells were well preserved (Figure 6A). In contrast, the organ of Corti in the Gentamicin group was severely lesioned (Figure 6B). In some areas, outer hair cells were entirely absent, and the inner hair cell population was partly depleted.

In the inner hair cell region, the number of scars in the Rescue group animals was significantly lower than that found in the Gentamicin group (Figure 7). In the outer hair cell region, a significant difference in the number of scars was observed between the Gentamicin, Ad.LacZ/Gentamicin and Rescue groups. In the Protection group, all animals had better preservation of cochlear hair cells than Gentamicin group animals. However, variability in the extent of cochlear hair cell loss was evident (Figure 7). Inner hair cell loss in the Protection group was significantly greater that that found in the Rescue group. There was a trend for more outer hair cell loss in the Protection group cochleae as compared with
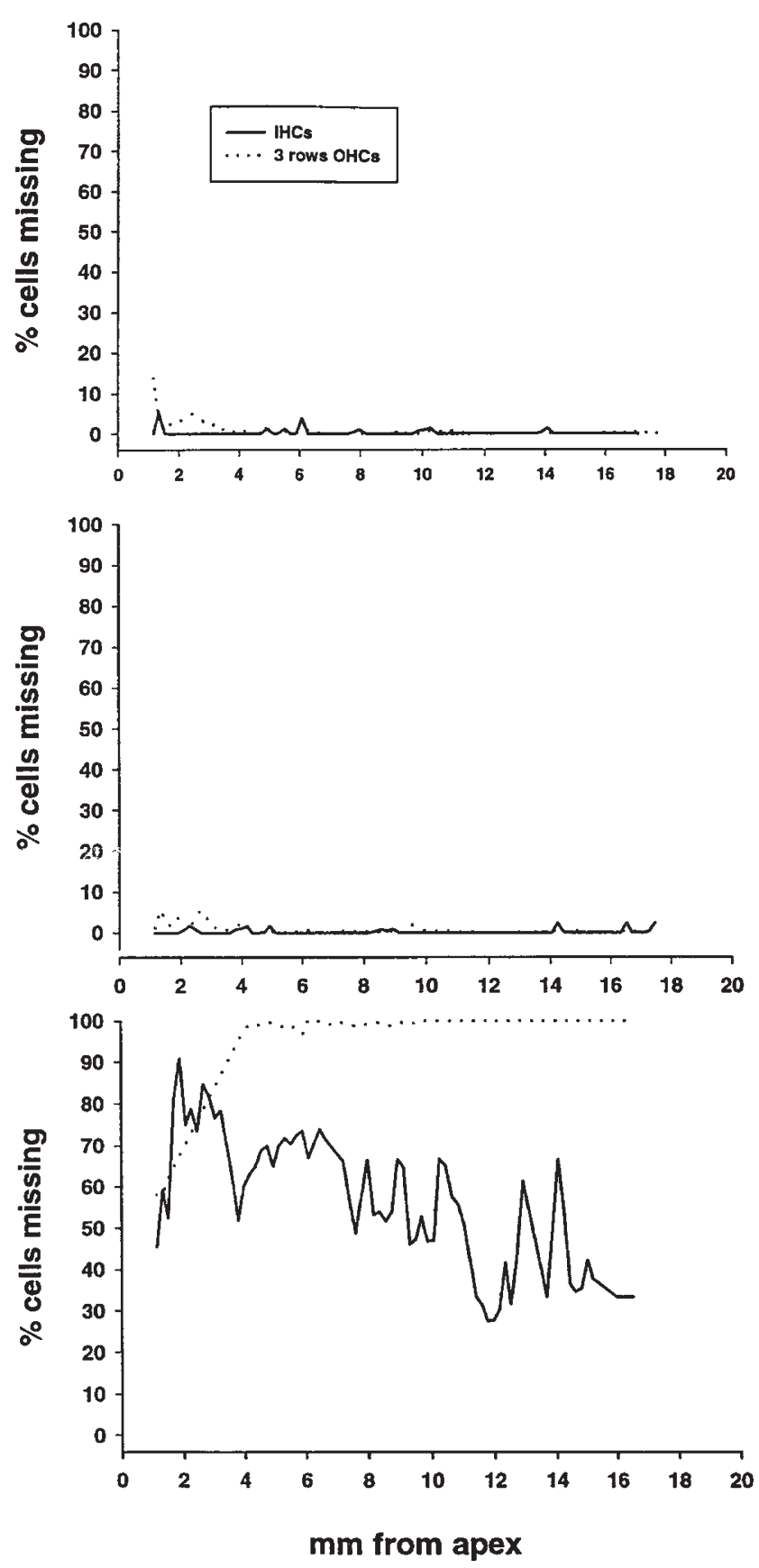

Figure 4 Mean cytocochleograms in Vehicle Control (top), Ad.LacZ Control (middle) and Gentamicin groups (bottom). The \% of hair cells missing is plotted as a function of their location along the cochlear duct ( $\mathrm{mm}$ from the apical end).

the Rescue group; however, the difference was not significant (Figure 7).

Both the inner and outer hair cell data failed to meet the normality criterion for parametric testing (criterion $P=0.05$ ). Nonparametric testing (ANOVA on ranks, Dunn's method of multiple pairwise comparison) indicated that the only statistically significant difference between groups was between the Rescue group and the Gentamicin group, for both IHC and OHC survival. It is important to keep in mind, however, that nonparametric tests are relatively conservative and so may overestimate the $P$ values. 

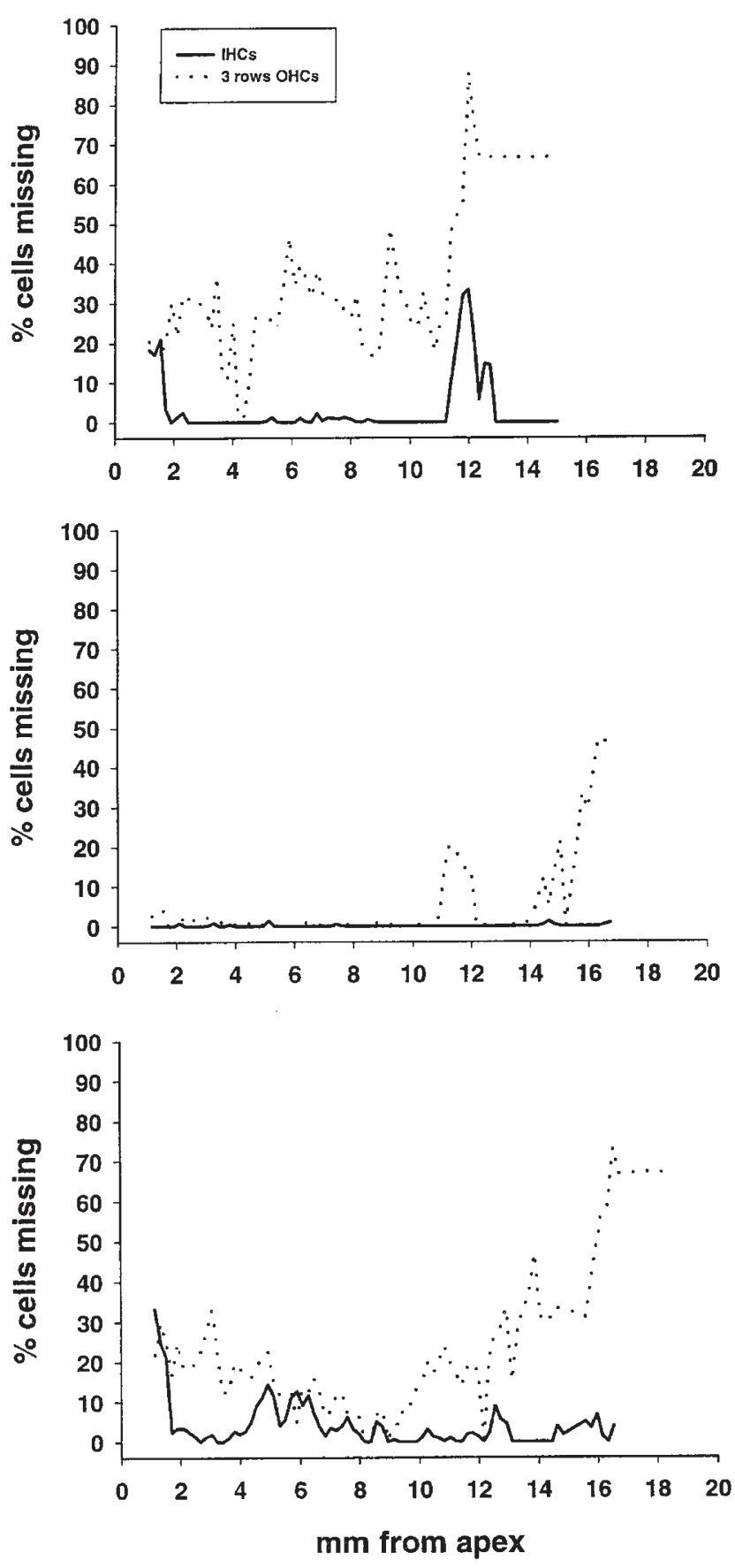

Figure 5 Mean cytocochleograms in Ad.LacZ/Gentamicin (top), Rescue (middle) and Protection (bottom) groups. The \% of hair cells missing is plotted as a function of their location along the cochlear duct ( $\mathrm{mm}$ from the apical end).

\section{Hair cell damage in the utricular macula}

No scars were observed in the utricular macula in the Vehicle or Ad.LacZ Control groups. A severe lesion in the utricular neuro-epithelium was observed in the striola portion in the Gentamicin and Ad.LacZ/Gentamicin groups, and in one animal in the Rescue group. In the Gentamicin and Ad.LacZ/Gentamicin groups, each animal showed numerous scars, which were composed of the expanded processes of the neighboring supporting cells in the reticular lamina of the utricular macula (Figure 6).
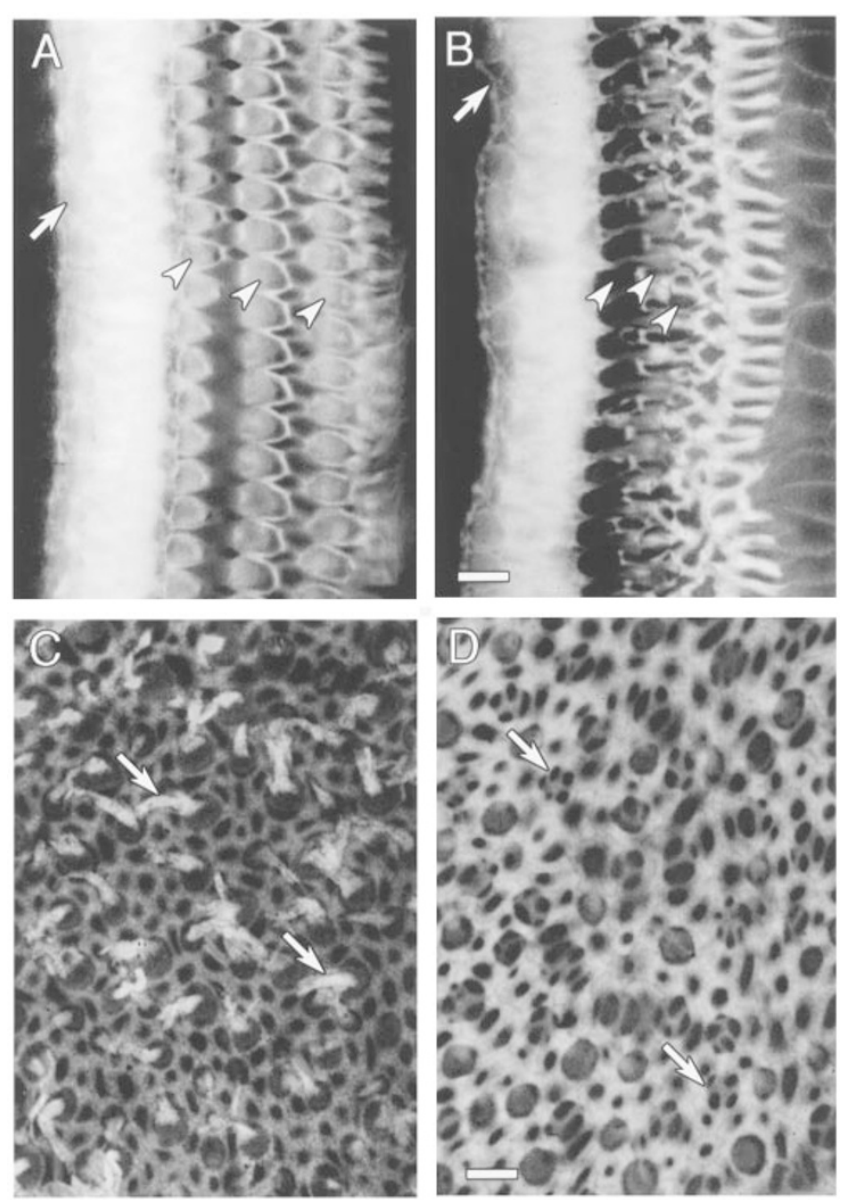

Figure 6 Whole-mounts of cochlear (A and B) and macular (C and D) tissue stained with rhodamine phalloidin and observed with epi-fluorescence. (A) The organ of Corti in the Rescue group. Inner (arrow) and outer hair cells (arrowheads) are well preserved. No scars are observed in the organ of Corti. (B) The organ of Corti in the Gentamicin group. Outer hair cells are entirely absent, replaced by scars (arrowheads). Several inner hair cells are also missing (arrow). (C) Utricular macula in the Protection group. The hair cells display normal-appearing stereocilia bundles (arrows). Supporting cells are normally arranged. (D) Utricular macula in the Gentamicin group. Several missing hair cells are replaced by scars, which are composed of expanded processes of three to five neighboring supporting cells (arrows). Bars, $10 \mu \mathrm{m}$ in (B) (for A and B) and $25 \mu \mathrm{m}$ in (D) (for C and D).

The degree of utricle scarring (scars $/ \mathrm{mm}^{2}$ ) was compared between groups using a one-way repeated measures analysis of variance, followed by a Student-Newman-Keuls multiple pairwise comparison. There was a statistically significant $(P<0.05)$ decrease in utricular scarring for both the Protection and Rescue groups, relative to the Gentamicin group (Figure 8). In addition, there was significantly less scarring in the Protection group, relative to both the Ad.LacZ/Gentamicin and Rescue groups.

\section{Discussion}

The results of this study show that Ad.GDNF can protect cochlear and vestibular hair cells from gentamicin ototoxicity. Cochlear function is also protected. The data demonstrate that the procedure for gene therapy in the inner ear is associated with mild side-effects, including a transi- 


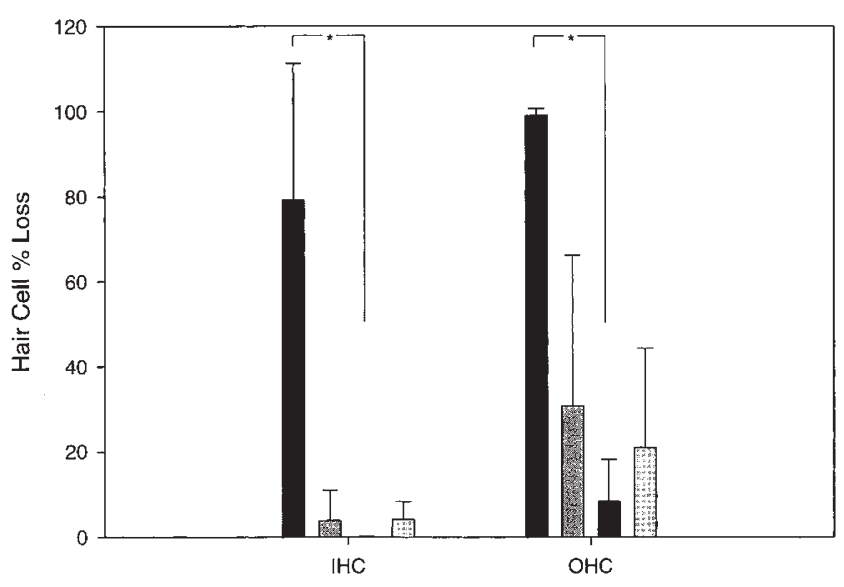

Gentamicin $(n=4)$ Ad.LacZ/Gentamicin $(n=6)$

Rescue $(n=8)$ Protection $(n=6)$

Figure 7 Average percent of inner and outer hair cell loss (mean) observed among the Gentamicin, Ad.LacZ/Gentamicin, Rescue and Protection groups. For both inner and outer hair cells, a significant difference is observed between the Gentamicin and Rescue groups. There is an obvious trend towards greater hair cell survival in the Protection and Ad.LacZ/Gentamicin groups, as well, relative to the Gentamicin group.

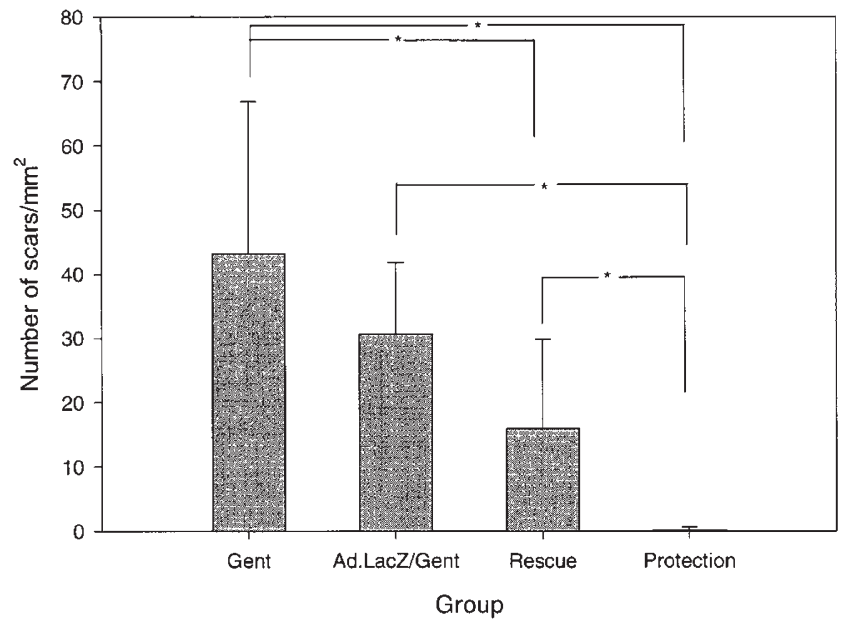

Figure 8 Mean number \pm s.d. of scar density in the utricular macula observed among the Gentamicin, Ad.LacZ/Gentamicin, Rescue and Protection groups. There is a significant decrease in scar density in the Protection group, relative to all other groups. In addition, there is a significant decrease in scar density in the Rescue group, relative to the Gentamicin group. *Significant difference $(P<0.05)$.

ent head tilt and, in some animals, a mild-to-moderate threshold shift at $20 \mathrm{kHz}$.

Intratympanic administration of gentamicin was selected as the means for inducing ototoxic trauma. This intervention is currently used in the clinical treatment of Meniere's disease. Since the most severe side-effect of gentamicin is renal toxicity, we chose an ear-specific application of gentamicin to prevent renal effects. ${ }^{23}$ Gentamicin administration into the middle ear has been shown to result in its presence in the organ of Corti and the vestibular neuro-epithelium. ${ }^{25}$ Gentamicin in the middle ear has also been shown to destroy hair cells. ${ }^{5}$
Following intratympanic application of aminoglycoside, hair cell degeneration in the cochlear and vestibular sensory epithelium has been found at 6 and 7 days, respectively. ${ }^{26,27}$ Therefore, we examined the tissue 7 days after gentamicin administration into the middle ear.

The results indicate that adenoviral vectors may be used in the inner ear to introduce genes that can protect hearing. Adenovirus-mediated gene transfer has some important advantages for the transfer and expression of therapeutic genes in the inner ear. Adenoviruses can infect quiescent or terminally differentiated cells. They can be prepared at high titers, allowing high efficiency transgene expression. Since adenovirus genomes generally do not integrate into the host cell chromosomes, insertional mutagenesis may be limited. ${ }^{28}$ Gene transfer experiments in the inner ear have been performed via direct inoculation of adenovirus vectors into the scala tympani, 19,21,29 because the blood-labyrinth barrier prevents access of adenoviral particles from the systemic circulation into the labyrinth. ${ }^{24}$

Several authors have described the distribution of transduced cells following inoculation of adenovirus vectors into the inner ear. The most efficiently transduced cells have been the mesothelial cells lining the perilymphatic space. ${ }^{21,24}$ Our data corroborate previous studies on the distribution of transduced cells in the inner ear. The cells that are most efficiently transduced with the Ad.GDNF vector most likely represent a population of cells similar to those transfected by the reporter gene. These cells are presumed to synthesize excess GDNF and secrete it, making it available to other cells in the inner ear.

Because of the location of the transduced cells in the inner ear, it is likely that GDNF was secreted into the perilymph. The pharmaco-kinetics of GDNF in the fluid compartments of the inner ear has not been determined; however, to influence cochlear and vestibular hair cells, GDNF most likely gained access to the vicinity of the hair cells. GDNF receptor localization on hair cells is not currently known. It is therefore unclear if the critical access is to the apical surface of the hair cells, which is bathed in endolymph, or the baso-lateral region, which is surrounded with fluid that is continuous with the scala tympani. Better cellular localization of the GDNF receptors as well as understanding of the spread of GDNF within the inner ear are necessary to explain the distribution and mechanism of action of the GDNF transgene.

Our choice of inoculation site, the scala vestibuli, was based primarily on the access it provides for the inoculated material into both the cochlea and the vestibular organs. In addition to accessibility issues, safety should also be an important consideration in choosing the site for inoculation. In the Vehicle Control group, no marked damage was observed morphologically in the cochlea and utricle, indicating that artificial perilymph administration into the scala vestibuli is safe for the hair cells in the cochlea and the utricle. These findings also indicate that the surgical procedure by itself is relatively benign. In the Ad.LacZ Control group, morphological changes in the cochlea were minimal but hearing thresholds were significantly elevated at $20 \mathrm{kHz}$, suggesting that the presence of the vector leads to some hearing impairment. Utricular macula morphology was unchanged in this group. These findings indicate that the area with the highest number of transduced cells, the base of the coch- 
lea, which is likely also to have the highest concentration of transgenic GDNF, tends to exhibit toxicity. It therefore appears that apart from an elevated threshold at $20 \mathrm{kHz}$, the Ad.LacZ Control group animals did not have a major functional deficit or significant pathology. For optimizing virus-mediated gene therapy for clinical use, it will be necessary to identify a viral titer that will result in minimal toxicity yet provide the expected beneficial effects.

Hair cell counts and thresholds measured in the Ad.LacZ/Gentamicin group were better than those in Gentamicin group, suggesting that Ad.LacZ inoculation can protect inner and outer hair cells from the damage induced by gentamicin. A similar trend for protection was afforded by the Ad.LacZ vector against kanamycin and ethacrynic acid cochleotoxicity. ${ }^{21}$ The protective effects of the Ad.LacZ vector are unexpected and deserve further consideration. Adenovirus-mediated gene transfer has been shown to induce an immune response in the sites of transfection. ${ }^{16}$ A mild proliferation of T lymphocytes has been observed in cochleae inoculated with the Ad.LacZ vector. ${ }^{19}$ In other tissues, an increase in the population of $\mathrm{T}$ cells or cytokines has been shown to upregulate the expression of neurotrophins, such as nerve growth factor (NGF), BDNF and NT-3, as well as GDNF. ${ }^{30-32}$ The relationship between inflammatory reactions and production of growth factors has been demonstrated in a variety of systems and species as well as in cultured cells. ${ }^{33,34}$ We speculate that the immune response due to Ad.LacZ might elevate the level of growth factors in the inner ear, leading to the protection of hair cells against aminoglycoside-induced ototoxicity. We observed a large variability in the extent of protection induced by Ad.GDNF inoculated 7 days before the gentamicin treatment. This variability may be a result of varying degrees of immune response.

The mechanism of action of GDNF in the protection of cochlear and vestibular hair cells and inner ear function is not completely understood. One possible mechanism is based on the mode of ototoxicity of gentamicin. Aminoglycoside-induced ototoxicity is associated initially with binding to phosphatidylinositol-4, 5bisphosphate, leading later to the formation of iron compounds with oxidative properties, promoting the formation of free radicals. ${ }^{35}$ GDNF has been shown to prevent free radical release and cortical infarction by the inhibition of neuronal free radical synthesis during ischemia. ${ }^{36}$ We speculate that GDNF may inhibit the free radical synthesis and release promoted by the redoxactive gentamicin compound, thus protecting cochlear and vestibular hair cells from the cytotoxic damage induced by free radical elevation. Alternatively, increased concentrations of GDNF in the inner ear neuroepithelia may cause direct protective effects by inhibiting cell death in these tissues. Finally, an indirect activity is possible, via up-regulating overexpression of other neurotrophic factors. For instance, it has been reported that the TGF- $\beta$ superfamily can up-regulate the production of fibroblast growth factor receptor mRNA in the mature spiral ganglion. ${ }^{37}$

The temporal sequence of gentamicin administration and GDNF transgene overexpression appears to be important in the determination of the extent of protection. The vector was inoculated into the perilymph at the lower part of the cochlear basal turn. With this approach, rescue of the lower basal turn and the utricular macula was incomplete, and ABR threshold shifts at $20 \mathrm{kHz}$ were greater than those measured at lower frequencies. The reason for the incomplete rescue in the lower base may be related to the presence of the highest concentration of viral vector particles in the basal turn, which could lead to cytotoxicity in this region. This idea is supported by the threshold shift recorded at $20 \mathrm{kHz}$ in the Ad.LacZ Control group.

In addition to the ability to prevent hair cell trauma by protection, clinical practice could benefit from the ability to rescue hair cells by intervention after the occurrence of insult. It has been demonstrated that BDNF can afford both rescue and protection of hair cells in the cristae from damage caused by gentamicin. ${ }^{5}$ Ad.GDNF can also be used to rescue cochlear and vestibular hair cells. Using adenoviral vectors, transcription has been reported to be activated approximately $8 \mathrm{~h}$ after infection following the onset of viral DNA synthesis. ${ }^{28}$ Gentamicin concentration is already near its peak level in the perilymph $8 \mathrm{~h}$ following inoculation into the middle ear. ${ }^{38}$ Therefore, the preservation of hair cells observed in the Rescue group is considered a rescue from the ototoxic insult. To advance further the understanding of the rescue capability of Ad.GDNF against ototoxic insults, it will be necessary to determine the latest stage in the pathological sequence at which rescue can be accomplished.

It is also important to determine the duration of protection afforded by the Ad.GDNF vector following inoculation. Since transduced cells can be detected 8 weeks after Ad.LacZ inoculation into the inner ear, ${ }^{39}$ it is likely that when using the GDNF vector, GDNF overexpression may last for many weeks after inoculation. The data on hair cell preservation in the Protection group indicate the continued protective effects afforded by Ad.GDNF 1 week after inoculation. Intuitively, protection should be more efficient than rescue, since the protective agent is present in the inner ear fluids at the time the insult is introduced. Results in the vestibular neuro-epithelium support this expectation, showing significantly better protection than rescue. However, in the cochlea, rescue was better than protection. To explain this finding, we speculate that elevated levels of GDNF can be achieved in the cochlea faster than in the vestibular organs. Therefore, several days of transgene expression are necessary to yield sufficiently high levels of transgene to allow for optimal protection of the utricular hair cells. To understand better the difference between rescue and protection, it will be necessary to characterize the spread of the virus and the secreted transgene in the inner ear fluids, and to elucidate the mechanisms of cell death in the vestibular and cochlear neuro-epithelium. It will also be important to determine if the immune response differs based on the timing of intervention. Regardless of the reasons for the difference between protection and rescue, the findings are of clinical importance, since they suggest that the rescue paradigm may be used preferentially to preserve cochlear hair cells, as discussed below.

In the clinic, intratympanic administration of gentamicin has been used as treatment for intractable unilateral Meniere's disease. ${ }^{40-43}$ This treatment is thought to be useful to control the vertigo caused by Meniere's disease, but an occurrence rate of gentamicin-induced hearing loss is reported to be $15-45 \% .{ }^{43}$ A sudden profound sensorineural hearing loss has occurred in about $10 \%$ of the patients after the intra-tympanic administration of 
gentamicin. $^{42,44}$ It is difficult to predict which patients will develop sensorineural hearing loss after such treatments. ${ }^{44}$ Therefore, it may be useful to actively protect the cochlea during the treatment aimed at eliminating vestibular hair cells. To achieve such selective vestibular lesions, it may be possible to restrict GDNF overexpression to cochlear areas. This may be possible by inoculation of Ad.GDNF into the scala tympani of the basal turn, which is the cochlear site most distant from the vestibular organs. Further studies are necessary to determine the optimal site of inoculation and the best temporal sequence for the administration of gentamicin and Ad.GDNF to obtain vestibular-restricted lesions.

\section{Materials and methods}

\section{Animals}

Thirty-two pigmented guinea pigs (outbred NIH strain, purchased from Murphy, Plainfield, IL, USA) with normal Preyer's reflexes weighing 300-450 g b.w. were used. The animals were divided into six groups:

(1) Vehicle Control group; artificial perilymph administration $(n=4)$.

(2) Ad.LacZ Control group; Ad.LacZ administration $(n=4)$.

(3) Gentamicin group; artificial perilymph followed immediately by gentamicin administration $(n=4)$.

(4) Ad.LacZ/Gentamicin group; Ad.LacZ followed immediately by gentamicin administration $(n=6)$.

(5) Rescue group; Ad.GDNF followed immediately by gentamicin administration $(n=8)$.

(6) Protection group; Ad.GDNF followed 7 days later by gentamicin administration $(n=6)$.

The experimental protocol used in this study was approved by the University of Michigan Committee for the Use and Care of Animals.

\section{Surgical procedures, viral inoculation and deafening}

Gentamicin vials $(40 \mathrm{mg} / \mathrm{ml})$ were purchased from Abbott Laboratories (North Chicago, IL, USA). Ad.LacZ and Ad.GDNF vectors were purchased from the Vector Core of the University of Michigan Center for Gene Therapy. Stock vector solution $\left(10^{10}\right.$ adenoviral particles $\left./ \mathrm{ml}\right)$ was diluted 1:2 in artificial perilymph $(\mathrm{pH}=7.42$, osmotic pressure $=290$ mosm) immediately before being used.

Animals were anesthetized with Rompun (xylazine, $10 \mathrm{mg} / \mathrm{kg}$; Bayer, Shawnee Mission, KS, USA) and Ketaset (ketamine-HCl, $40 \mathrm{mg} / \mathrm{kg}$; Parke Davis, Morris Plains, NJ, USA). An incision was made in the left submandibular region. The left tympanic bulla was exposed via the ventral approach, leaving the styloid process and the posterior belly of the digastric muscle intact. A small hole was made in the tympanic bulla to expose the lateral wall of the cochlear basal turn. A small fenestra was then made on the lateral wall of the scala vestibuli in the cochlear basal turn using a custom-made needle. Four $\mu l$ of either artificial perilymph $(n=8)$, Ad.LacZ $(n=10)$ solution or Ad.GDNF $(n=14)$ solution were administered into the scala vestibuli through the fenestra, using a $10 \mu \mathrm{l}$ Hamilton syringe with a 35-gauge needle. The fenestra was sealed with fascia of the sternocleidomastoideus muscle and bone chips immediately after the administration.
To induce ototoxic trauma, gentamicin was administered into the middle ear cavity of the left ear. Using this procedure, renal damage could be avoided. The tympanic cavity was filled with about $200 \mu$ l gentamicin solution 5 min after the closure of the fenestra on the cochlear lateral wall. Then, the bony defect of the tympanic bulla was sealed with Durelon (ESPE, Norristown, PA, USA), and the skin was closed with suture.

In Protection group animals, the bony defect in the tympanic bulla was sealed with Durelon minutes after the closure of the fenestra on the lateral wall. Next, the skin was closed with sutures. Seven days later, the left temporal bone was perforated using the postauricular approach and the tympanic cavity was filled with about $200 \mu \mathrm{l}$ of gentamicin solution under general anesthesia with Rompun and Ketaset. The bony defect was sealed with Durelon and the skin closed with sutures. Animals in the Vehicle Control group and Ad.LacZ Control group were not injected with gentamicin.

After surgery, chloramphenicol sodium succinate (30 mg/kg) was administered intramuscularly as prophylaxis. Animals were weighed and head tilt evaluated daily until decapitation.

\section{Monitoring for hearing function}

Hearing thresholds were measured in left ears with pure tone ABRs at 4, 12 or $20 \mathrm{kHz}$ before surgery (to establish baseline) and on the day of decapitation. ABRs were performed in all animals in the Vehicle Control, Ad.LacZ Control, Gentamicin and Rescue groups, and in five of the six animals in the Ad.LacZ/Gentamicin group. Measurements in one animal in the Ad.LacZ/Gentamicin group and in all animals in the Protection group were not performed due to technical reasons. ABRs were measured under general anesthesia with intramuscular injection of a solution of Rompun and Ketaset. All animals were placed on a heated pad and covered with a blanket to maintain the animal's core temperature at $38^{\circ} \mathrm{C}$. The active electrode was placed on the scalp behind the left ear, the indifferent electrode on the frontal region of the head, and the ground on the scalp behind the right ear. The stimulus consisted of $15 \mathrm{~ms}$ tone bursts, with a risefall time of $1 \mathrm{~ms}$. Near the threshold, responses to 1024 sweeps were averaged at each intensity level. Levels were decreased in $5 \mathrm{~dB}$ steps. The threshold was defined as the lowest level at which waves P3 or P4 of the ABR could be detected.

\section{Tissue preparation}

All guinea pigs were anesthetized and killed by decapitation 7 days after the last surgery. The left bony labyrinth was extirpated (the right ears were not used in this study). The stapes were removed, and the cochlear apex and round window membrane were perforated. The inner ear was briefly rinsed with PBS solution and fixed with cold $4 \%$ paraformaldehyde for $3 \mathrm{~h}$. After fixation, tissues of Ad.LacZ administered animals were rinsed with PBS and incubated over night in 5-bromo-4-chloro3-indolyl- $\beta$-D-galactopyranoside $(X$-gal), rinsed in PBS and observed under stereoscopic magnification.

In all animals, the lateral wall, tectorial membrane and Reissner's membrane were removed, and the modiolus with the organ of Corti was detached from the temporal bone. The utricle was then removed from the bony labyrinth. The modiolus with the organ of Corti and utricle 
was immersed in $0.3 \%$ Triton-X-100 in PBS for $10 \mathrm{~min}$ then incubated for $30 \mathrm{~min}$ at room temperature in rhodamine phalloidin (Molecular Probes, Eugene, OR, USA) diluted 1:100 in PBS. After a PBS rinse, the organ of Corti was separated from the modiolus in half-turn segments, and the membranes around the utricular macula were removed, along with the otolithic membrane. The organ of Corti and the utricle were mounted on glass slides with Crystal/Mount (Biomeda, Foster City, CA, USA) and examined and photographed using a Leica (Eaton, PA, USA) DMRB epi-fluorescence microscope.

\section{Data analysis}

To evaluate physiological changes of the cochlea, ABR threshold shifts were calculated in each animal by comparing the thresholds just before decapitation with their baselines. The upper limit of sound pressure was set at $85 \mathrm{~dB}$ SPL. When ABR threshold levels were beyond this level, threshold was assumed to be $90 \mathrm{~dB}$ SPL, which presumably yields a conservative measure of the threshold shift.

Morphological changes in the cochlea were quantified with fluorescence microscopy. The number of outer and inner hair cells was counted from the apex to the base as previously described. ${ }^{21}$ Counting was begun at $2 \mathrm{~mm}$ from the apex, and completed at $18 \mathrm{~mm}$ from the apex, at the middle of the hook region. In some cases a small segment of the whole-mount preparation was imperfect, making counting unreliable or impossible. Data from these segments were excluded, from the graphs and the statistical analysis. To generate and plot cytocochleograms, we used customized Excel-based software, which compared the hair cell counts for each experimental animal with a normal count of cochlear hair cells obtained from normal guinea pigs of the same strain. The plots show the percentage of hair cell loss as a function of their location along the cochlear duct. For each ear, the normalized inner and outer hair cell loss was calculated for each measured tissue segment $(0.19 \mathrm{~mm}$ in length). Mean hair cell loss was then calculated for each group. For each segment, the means of at least three data points (one from each animal) had to be present for inclusion in the graph. However, in the Ad.LacZ Control group, means from two or more values were allowed because of localized dissection artifacts in two of the four ears.

Scars which are left in sites of hair cell loss in the utricular macula are composed of the expanded processes of the three to five neighboring supporting cells. ${ }^{45}$ Because hair cell damage due to aminoglycoside toxicity in the utricular macula is most severe in the striola, ${ }^{27}$ we chose to evaluate a portion of the utricle that included striolar tissue. A series of micrographs focused at the reticular lamina was taken in each animal using an epifluorescence microscope and slide (diapositive) photographic film. The images of four or five photographs, selected from each animal at random, were projected with a slide projector on a screen to facilitate scar counts. After counting the scars, the slides were digitized and imported to the MetaMorph software, which was used for measuring the area in which scar quantitation took place. The total number of scars in each image was summed for all images per animal and divided by the average area of measurement. This calculation yielded scar density (scar number $/ \mathrm{mm}^{2}$ ) for each animal. Then the means \pm s.d. of scar density were calculated for each experimental group.

Sigma Stat statistical software (Jandel; SPSS, Chicago, IL, USA) was used for statistical analysis. ABR threshold shifts, cochlear hair cell loss and scar density were compared using analyses of variance (ANOVA). When significant differences were found by ANOVA, multiple pairwise comparisons were made using the Tukey, Bonferroni, or Student-Newman-Keuls methods. In all cases, a value of $P<0.05$ was considered statistically significant.

\section{Acknowledgements}

We wish to thank Timo Stöver for helpful discussions and Joseph E Hawkins for valuable comments on the manuscript. AL Miller is funded by a Rackham Predoctoral Fellowship. This study was supported by NIHNIDCD grants PO1 DC00078 and R01 DC01634 (YR).

\section{References}

1 Shieh PB, Ghosh A. Neurotrophins: new roles for a seasoned cast. Current Biol 1997; 7: 627-630.

2 Ernfors P, Van De Water T, Loring J, Yaenisch R. Complementary roles of BDNF and NT3 in vestibular and auditory development. Neuron 1995; 14: 1153-1164.

3 Pirvola U et al. Brain-derived neurotrophic factor and neurotrophin 3 mRNAs in the peripheral target fields of developing inner ear ganglia. Proc Natl Acad Sci USA 1992; 89: 9915-9919.

4 Ylikoski J et al. Expression patterns of neurotrophin and their receptor mRNAs in the rat inner ear. Hear Res 1993; 65: 69-78.

5 Lopez I et al. The protective effect of brain-derived neurotrophic factor after gentamicin ototoxicity. Am J Otol 1999; 20: 317-324.

6 Lin LF et al. GDNF a glial cell line-derived neurotrophic factor from midbrain dopaminergic neurons. Science 1993; 260: 11301132

7 Buj-Bello A et al. GDNF is an age-specific survival factor for sensory and autonomic neurons. Neuron 1995; 15: 821-828.

8 Trupp $\mathrm{M}$ et al. Peripheral expression and biological activities of GDNF, a new neurotrophic factor for avian and mammalian peripheral neurons. J Cell Biol 1995; 130: 137-148.

9 Nosrat CA et al. Cellular expression of GDNF mRNA suggests multiple functions inside and outside the nervous system. Cell Tissue Res 1996; 286: 191-207.

10 Ylikoski J et al. Guinea pig auditory neurons are protected by glial cell line-derived growth factor from degeneration after noise trauma. Hear Res 1998; 124: 17-26.

11 Stöver $\mathrm{T}$ et al. Expression of the GDNF family members and their receptors in the mature rat cochlea. Mol Brain Res 2000; 76: 23-35.

12 Yamasoba T, Schacht J, Shoji F, Miller JM. Attenuation of cochlear damage from noise trauma by an iron chelator, a free radical scavenger and glial cell line-derived neurotrophic factor in vivo. Brain Res 1998; 815: 317-325.

13 Keithley EM et al. GDNF protects the cochlea against noise damage. Neuroreport 1998; 9: 2183-2187.

14 Nabel GJ. From rhyme to reason. Nature 1998; 392: 3-4.

15 Anderson WF. Human gene therapy. Nature 1998; 392 (Suppl.): 25-30.

16 Wilson JM. Molecular medicine: adenoviruses as gene-delivery vehicles. Mol Med 1996; 334: 1185-1187.

17 Staecker H, Gabaizadeh R, Federoff H, Van De Water TR. Brainderived neurotrophic factor gene therapy prevents spiral ganglion degeneration after hair cell loss. Otolaryngol Head Neck Surg 1998; 119: 7-13.

18 Holt JR et al. Functional expression of exogenous proteins in mammalian sensory hair cells infected with adenoviral vectors. J Neurophysiol 1999; 81: 1881-1888. 
19 Raphael Y, Frisancho JC, Roessler BJ. Adenoviral-mediated gene-transfer into cochlear cells in vivo. Neurosci Lett 1996; 207: 137-141.

20 Lalwani AK et al. Development of in vivo gene therapy for hearing disorders: introduction of AAV into the cochlea of the guinea pig. Gene Therapy 1996; 3: 588-592.

21 Yagi $\mathrm{M}$ et al. Hair cells are protected from aminoglycoside ototoxicity by adenoviral-mediated overexpression of GDNF. Hum Gene Ther 1999; 10: 813-823.

22 Matz GJ. Aminoglycoside cochlear ototoxicity. In: Rybak LP (ed). Ototoxicity, Otolaryngol Clin North Am. WB Saunders: Philadelphia, 1993, pp 705-712.

23 Hawkins JE, Johnsson L-G, Aran J-M. Comparative tests of gentamicin ototoxicity. J Infect Dis 1969; 119: 417-431.

24 Stöver T, Yagi M, Raphael Y. Transduction of the contralateral ear after adenoviral-mediated cochlear gene transfer. Gene Therapy 2000; 7: 377-383.

25 Hayashida T. An immunohistochemical method for the study of aminoglycoside ototoxicity in the guinea pig cochlea using decalcified frozen section. Arch Otorhinolaryngol 1989; 246: 161-164.

26 Wright CG, Meyerhoff WL. Ototoxicity of otic drops applied to the middle ear in the chinchilla. Am J Otolaryngol 1984; 5: 166-176.

27 Sera $\mathrm{K}$ et al. Morphological changes in the vestibular epithelia and ganglion induced by ototoxic drug. Scan Electron Microsc 1987; 1: 1191-1197.

28 Ali M, Lemoine NR, Ring CJA. The use of DNA viruses as vectors for gene therapy. Gene Therapy 1994; 1: 367-384.

29 Stöver T, Yagi M, Raphael Y. Cochlear gene transfer: round window versus cochleostomy inoculation. Hear Res 1999; 136: 124-130.

30 Saporito MS et al. Pharmacological induction of nerve growth factor mRNA in adult rat brain. Exp Neurol 1993; 123: 295-302.

31 Friedman WJ, Thakur S, Seidman L, Rabson AB. Regulation of nerve growth factor mRNA by interleukin-1 in rat hippocampal astrocytes is mediated by NFkappaB. J Biol Chem 1996; 271: 31115-31120.
32 Appel E et al. Regulation of GDNF expression in cultured astrocytes by inflammatory stimuli. Neuroreport 1997; 8: 3309-3312.

33 Oddiah D, Anand P, McMahon SB, Rattray M. Rapid increase of NGF, BDNF and NT-3 mRNAs in inflamed bladder. Neuroreport 1998; 9: 1455-1458.

34 Virchow JC et al. Neurotrophins are increased in bronchoalveolar lavage fluid after segmental allergen provocation. Am J Respir Crit Care Med 1998; 158: 2002-2005.

35 Schacht J. Aminoglycoside ototoxicity: prevention in sight? Otolaryngol Head Neck Surg 1998; 118: 674-677.

36 Wang Y et al. Glial cell line-derived neurotrophic factor protects against ischemia-induced injury in the cerebral cortex. J Neurosci 1997; 17: 4341-4348.

37 Lefebvre PP et al. TGFSS1 modulates bFGF receptor message expression in cultured adult auditory neurons. Neuroreport 1991; 2: 305-308.

38 Balough BJ et al. Kinetics of gentamicin uptake in the inner ear of Chinchilla langier after middle-ear administration in a sustained-release vehicle. Otolaryngol Head Neck Surg 1998; 119: 427-431.

39 Komeda M, Roessler JB, Raphael Y. The influence of interleukin1 receptor antagonist transgene on spiral ganglion neurons. Hear Res 1999; 131: 1-10.

40 Minor LB. Intratympanic gentamicin for control of vertigo in Meniere's disease: vestibular signs that specify completion of therapy. Am J Otol 1999; 20: 209-219.

41 Nedzelski JM et al. Intratympanic gentamicin instillation as treatment of unilateral Meniere's disease: update of an ongoing study. Am J Otol 1993; 14: 278-282.

42 Eklund $S$ et al. Effect of intratympanic gentamicin on hearing and tinnitus in Meniere's disease. Am J Otol 1999; 20: 350-356.

43 Monsell EM, Cass SP, Rybak LP. Therapeutic use of aminoglycosides in Meniere's disease. Otolaryngol Clin North Am 1993; 26: 737-746.

44 Hirsch BE, Kamerer DB. Role of chemical labyrinthectomy in the treatment of Meniere's disease. Otolaryngol Clin North Am 1997; 30: 1039-1049.

45 Meiteles LZ, Raphael Y. Scar formation in the vestibular epithelium after aminoglycoside toxicity. Hear Res 1994; 79: 26-38. 